\title{
Impact of inlet design on mass transfer in gas-liquid rectangular microchannels
}

\section{Journal Article}

Author(s):

Fries, Donata Maria; Rudolf von Rohr, Philipp

Publication date:

2009

Permanent link:

https://doi.org/10.3929/ethz-b-000010964

Rights / license:

In Copyright - Non-Commercial Use Permitted

Originally published in:

Microfluidics and Nanofluidics 6(1), https://doi.org/10.1007/s10404-008-0292-6 


\title{
Impact of inlet design on mass transfer in gas-liquid rectangular microchannels
}

\author{
Donata Maria Fries $\cdot$ Philipp Rudolf von Rohr
}

Received: 20 October 2007/ Accepted: 28 March 2008/Published online: 29 April 2008

(C) Springer-Verlag 2008

\begin{abstract}
The length of the gas bubbles as well as of the liquid slugs in Taylor flow in rectangular microchannels was studied. At constant flow ratios of the gas and the liquid phase, we were able to vary the unit cell length, and therefore the gas bubble length as well as the liquid slug length by factor 4 solely by changing the inlet geometry. Based on literature and experimental data, we state a new correlation to predict the gas bubble length, the liquid slug length as well as the pressure drop for rectangular microchannels. The observed decrease in slug length for the investigated case corresponds to an increase in the Sherwood number and therefore of the mass transfer by a factor of 1.6. Short unit cell lengths, meaning enhanced mass transfer within the liquid slug and increased interfacial area, can be achieved for given flow rates using a small gas inlet channel compared to the main channel and injection in flow direction.
\end{abstract}

Keywords Gas-liquid flow · Gas inlet · Pressure drop · Mass transfer · Unit cell length

\section{Introduction}

Multiphase flow is an important aspect in chemical engineering. In microreactors, the so-called Taylor flow is one of the most often used flow patterns. It is characterized by a continuous phase (liquid) preferably wetting the wall of the channel and a second non-soluble phase (gas or a second

D. M. Fries · P. Rudolf von Rohr $(\square)$

Institute of Process Engineering,

ETH Zurich, Sonneggstr. 3, 8092 Zurich,

Switzerland

e-mail:vonrohr@ipe.mavt.ethz.ch liquid), which forms bubbles or droplets with a diameter close to the channel diameter and a length between 2 and 20 channel diameters. These bubbles or droplets are interrupted by slugs of the continuous phase, usually with a length of about the channel diameter. Important parameters influencing the mass transfer are the length of both, the gas bubbles $\left(L_{\mathrm{B}}\right)$ and the liquid slugs $\left(L_{\mathrm{S}}\right)$ (see Fig. 1) as well as the thickness of the liquid film at the wall, see (Kreutzer et al. 2005a). For instance, the Sherwood number, characterizing the total mass transfer versus the mass transfer due to diffusion, increases by a factor of 1.5 while decreasing the dimensionless liquid slug length from 2 to 0.8 . The following correlation was found by Kreutzer et al. (2005a):

$S h \equiv \frac{k d}{D}=\sqrt{\alpha^{2}+\frac{\beta}{G z}}$

with

$$
\begin{aligned}
\alpha & =40\left(1+0.28\left(L_{\mathrm{S}} / d\right)^{-4 / 3}\right) \\
\beta & =90+104\left(L_{\mathrm{S}} / d\right)^{-4 / 3} \\
G z & =L_{\text {Channel }} /(d \operatorname{Re} S c)
\end{aligned}
$$

The Schmidt number $S c$ characterizes the fluid properties: $S c=\eta /(\rho D)$. According to this correlation, Sh can be increased in our specific case (Ethanol-Nitrogen) by a factor of 1.6 due to changes in the dimensionless slug length $L_{\mathrm{s}} / d$ from 2 to 0.5 .

Different literature sources state that the length of the gas bubbles and liquid slugs strongly depends on the gas inlet design. In this paper, we investigate different designs, varying the channel width $w$ at a constant channel height $h$, the ratio of $w$ to the width of the gas inlet $\gamma$ and the inlet angle $\beta$. 


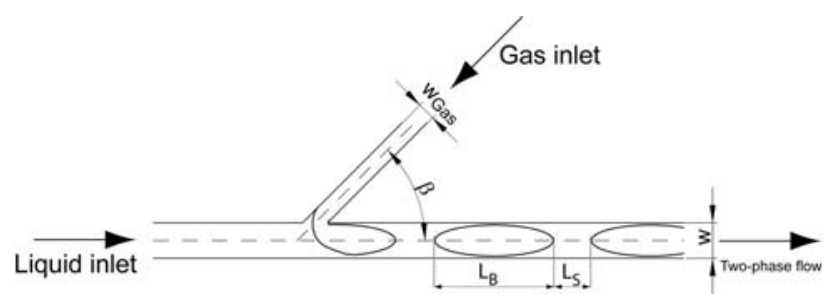

Fig. 1 Scheme of the used microreactors

\subsection{Gas bubble formation and length}

The defined formation of bubbles in microchannels is in the focus of recent research projects. First work was done using capillaries in the miniscale. Laborie et al. (1999) found

$\frac{L_{\mathrm{B}}}{d_{\mathrm{h}}}=0.0878\left(\frac{R e_{\mathrm{L}}\left(v_{\mathrm{B}}\right)}{B o^{2}}\right)^{0.63}$

to fit the measured data best. $d_{\mathrm{h}}$ is the hydraulic diameter of the capillary, $R e$ the Reynolds number defined as $R e=\left(\rho_{\mathrm{L}}\right.$ $\left.v_{\mathrm{B}} d_{\mathrm{h}}\right) / \eta_{\mathrm{L}}$ (where the subscript $L$ denotes the liquid phase, $\rho$ the density, $v_{\mathrm{B}}$ the intrinsic bubble velocity and $\eta$ the viscosity $). B o$ is the Bondnumber, calculated by $B o=\left[\left(\rho_{\mathrm{L}}-\right.\right.$ $\left.\left.\rho_{\mathrm{G}}\right) g d_{\mathrm{h}}^{2}\right] / \sigma$ with $\sigma$ as surface tension and $g$ as gravitational constant. In this correlation, important physical parameters as the inertia forces, viscosity forces and gravity forces as well as the channel diameter play a significant role. The geometry of the gas inlet has not been considered. As in microscale the gravity can be neglected, Eq. 2 has to be modified. Zheng and Che (2006) observed in a circular vertical pipe that increasing the superficial gas velocity as well as decreasing the superficial liquid velocity result in an increased gas bubble length and a shorter liquid slug length.

Garstecki et al. (2006) investigated the droplet length for liquid-liquid flow as well as for gas-liquid flow in microchannels depending on the flow rates. They highlighted a dependence of the breakup of the gas bubbles and thus of the gas bubble length via the pressure equilibrium. Assuming a pressure balance of the continuous (liquid) and the discontinuous (gas) phase showed a strong influence of the laplace pressure $\Delta p_{\mathrm{L}}=\sigma\left(\frac{1}{r_{1}}+\frac{1}{r_{2}}\right)$ with $r_{1}$ equal to half of the gas bubble height and $r_{2}$ the observed radius at the neck. At the beginning, the liquid phase is flowing and no gas enters the main channel. The gas phase has to overcome the resistance of the gas-liquid interface and of the liquid flow resistance. Therefore, the pressure in the gas phase increases. At a certain threshold value, depending on the system pressure, the gas phase enters the main channel. First, it has a spherical form, but as soon as the diameter of the gas circle is close to the channel width, the gas bubble "grows" towards the reactor outlet. During this procedure, the liquid phase is partially dammed, i.e. the liquid pressure increases. Note, that in rectangular channels as they had been used in this study a certain amount of liquid can pass in the corners, see van Steijn et al. (2007). Due to this pressure, the elongated gas bubble will be shortened on the neck so that less gas can flow. At a certain neck thickness (i.e. a maximum of the Laplace pressure), the gas flow is interrupted and the liquid flows again. This process is repeated continuously.

The discontinuous phase is injected into the continuous via a smaller channel, merging into the main channel at a right angle. The width of the main channel varied between 50 and $200 \mu \mathrm{m}$, the height was 33 and $79 \mu \mathrm{m}$, respectively. The width of the inlet channel for the disperse phase was 50 and $100 \mu \mathrm{m}$, respectively. As characteristic length they used the droplet length divided by the main channel width. Using a simple scaling law leads to:

$\frac{L_{\mathrm{B}}}{w}=1+\alpha \frac{\dot{V}_{\mathrm{G}}}{\dot{V}_{\mathrm{L}}}$

where $w$ represents the width of the main channel, $\alpha$ a geometry-dependent constant in the order of one and $\dot{V}_{\mathrm{G}}$ and $\dot{V}_{\mathrm{L}}$ the volume flow rates of the gaseous and the liquid phase. This model did not fit for gas-liquid flow at higher gas flow rates $\left(\dot{V}_{\mathrm{G}}>\dot{V}_{\mathrm{L}}\right)$, independent of the channel width.

\subsection{Liquid slug length}

In this context the liquid slug length in microchannels is of special interest: Since a strong recirculation occurs in these slugs (Waelchli and Rudolf von Rohr 2006; King et al. 2007), short slugs are advantageous for a strong mass transfer and mixing. Furthermore, Bercic and Pintar (1997) demonstrated that short slugs result in a higher interfacial area, which is also advantageous for enhanced mass transfer. For circular capillaries, Laborie et al. (1999) found

$\frac{L_{\mathrm{S}}}{d_{\mathrm{h}}}=3451\left(\frac{1}{\operatorname{Re}_{\mathrm{L}}\left(j_{G}\right) B o}\right)^{1.2688}$

with $j_{G}$ as the superficial velocity to fit the measured data best. Heiszwolf et al. (2001) correlated the dimensionless slug length $L_{\mathrm{S}} / d_{\mathrm{h}}$ in a monolithic structure to the unit cell $\left(L_{\mathrm{S}}+L_{\mathrm{B}}\right) / d_{\mathrm{h}}$ length via the liquid holdup $\epsilon_{\mathrm{L}}=V_{S} /\left(V_{S}+V_{G}\right)$.

$\frac{L_{\mathrm{S}}}{d_{\mathrm{h}}}=\epsilon_{\mathrm{L}} \frac{L_{\mathrm{S}}+L_{\mathrm{B}}}{d_{\mathrm{h}}}$

They observed a constant dimensionless liquid slug length $L_{\mathrm{S}} / d_{\mathrm{h}}$ of 2 for typical holdup values in the range of $\epsilon_{\mathrm{L}}=$ $0.2, \ldots, 0.5$. Based on these results, they obtained the following correlation: 
$\frac{L_{\mathrm{S}}}{d_{\mathrm{h}}}=\frac{\epsilon_{\mathrm{L}}}{-0.00141-1.556 \epsilon_{\mathrm{L}}^{2} \ln \left(\epsilon_{\mathrm{L}}\right)}$

The effect of the channel size for monolithic reactors has been investigated by Yawalkar et al. (2005), demonstrating that larger capillaries lead to elongated slugs. Tice et al. (2003) showed that in a rectangular microreactor for liquid-liquid two phase flow the unit cell length as well as the droplet length are independent of the total flow velocity but depend strongly on the flow rates of both phases. In their experiments, the droplet length fitted well with Eq. 5, using the volumetric flow ratio: $\epsilon_{\mathrm{L}}^{*}=\dot{V}_{\mathrm{L}} /\left(\dot{V}_{\mathrm{L}}+\dot{V}_{\mathrm{G}}\right)$. They also found that mixing within the slug is faster for short slug lengths. Liu et al. (2005) pointed out that for given flow rates the resulting liquid slug length in a circular or rectangular capillary also depends on the inlet geometry. For their nozzle geometry, they obtained

$L_{\mathrm{S}}=\left[\frac{j_{\mathrm{L}}+j_{\mathrm{G}}}{0.088 R e_{\mathrm{G}}^{0.72} R e_{\mathrm{L}}^{0.19}}\right]^{2}$

Akbar and Ghiaasiaan (2006) used the volume of fluid method to predict the liquid slug length in circular capillaries and compared the results with experimental data.

$\frac{\left(j_{\mathrm{L}}+j_{\mathrm{G}}\right)^{-0.33}}{\sqrt{L_{\mathrm{S}}}}=142.6 \epsilon_{\mathrm{G}}^{0.56}\left(\frac{d_{\mathrm{h}}}{L_{\mathrm{S}}+L_{\mathrm{B}}}\right) R e_{\mathrm{G}}^{-0.252}$

with $\epsilon_{\mathrm{G}}$ as gas holdup. Note that Eqs. 5 and 7 require knowledge of the unit cell length $L_{\mathrm{S}}+L_{\mathrm{B}}$ to predict the slug length. In contrast to these correlations where no influence of the inlet design had been considered, we propose in this contribution a significant impact of the inlet design on the gas-liquid flow characteristic in rectangular microchannels.

\section{Experimental setup}

Microreactors have been fabricated using standard softlithography: a master was fabricated by patterning a $100 \mathrm{~mm}$-wafer with photolithographical methods (SU-8). This master was molded with Polydimethylsiloxane (PDMS), which had been cured at $70^{\circ} \mathrm{C}$ for $4 \mathrm{~h}$ in an oven. After peeling off, the PDMS-reactors were cut, and the inlet and outlet holes were punched. Then the surfaces of the reactor and the cover glass were activated in an oxygen plasma and sealed together. Finally, inlet and outlet tubings were glued (McDonald 2002).

A total of 24 reactor designs, varying in channel width $w=200,300,400 \mu \mathrm{m}$, gas inlet angle $\beta=45^{\circ}, 90^{\circ}, 135^{\circ}$ and the ratio of the width of the gas inlet and the channel diameter $\gamma=w_{\mathrm{Gas}}: w=1: 1, \quad 1: 2, \quad 1: 3$ were used (see Fig. 1). In Table 1 all reactors are characterized and named
Table 1 Design parameters of the used reactors

\begin{tabular}{lllll}
\hline$\beta$ & $\gamma$ & $\begin{array}{l}w=200 \mu \mathrm{m} \\
h=150 \mu \mathrm{m}\end{array}$ & $\begin{array}{l}w=300 \mu \mathrm{m} \\
h=160 \mu \mathrm{m}\end{array}$ & $\begin{array}{l}w=400 \mu \mathrm{m} \\
h=230 \mu \mathrm{m}\end{array}$ \\
\hline $90^{\circ}$ & $1: 1$ & $90-200-1$ & $90-300-1$ & $90-400-1$ \\
& $1: 2$ & $90-200-2$ & $90-300-2$ & $90-400-2$ \\
& $1: 3$ & $90-200-3$ & $90-300-3$ & $90-400-3$ \\
$45^{\circ}$ & $1: 1$ & $45-200-1$ & $45-300-1$ & $45-400-1$ \\
& $1: 2$ & $45-200-2$ & $45-300-2$ & $45-400-2$ \\
& $1: 3$ & $45-200-3$ & $45-300-3$ & $45-400-3$ \\
$135^{\circ}$ & $1: 1$ & $135-200-1$ & $135-300-1$ & - \\
& $1: 2$ & $135-200-2$ & $135-300-2$ & - \\
& $1: 3$ & $135-200-3$ & $135-300-3$ & - \\
\hline
\end{tabular}

with the term used in the following text. Although a $\beta=90^{\circ}$ inlet is a commonly used design, the other angles had been chosen arbitrarily.

The liquid phase (Rhodamine B-colored (Fluka) Ethanol), was delivered by a syringe pump (Harvard Apparatus, PHD 2000). The gas phase (nitrogen) was controlled via mass flow controllers (Bronkhorst). The experiments were performed with $R e=15, \ldots, 40$ and $W e=0.3, \ldots, 0.7$. To characterize the phases, we used Laser Induced Fluorescence (LIF). The microreactor was positioned at an inversed microscope (Zeiss). The Nd:YAG-laser (NewWave) with an excitation wavelength of $532 \mathrm{~nm}$ and the camera (LaVision) were triggered by a synchronizer (LaVision). Double-exposed, double-framed pictures were taken with a frequency of $5 \mathrm{~Hz}$. The field of view was $1,090 \mu \mathrm{m} \times 1,300 \mu \mathrm{m}$. Optical measurements were done $30 \mathrm{~mm}$ behind the gas inlet and $15 \mathrm{~mm}$ before the outlet. Pictures were analyzed using Matlab. The pressure drop was measured by pressure transducers at the liquid inlet and the outlet (Endress and Hausser). The temperature was kept constant at $T=20^{\circ} \mathrm{C}$.

\section{Results}

\subsection{Gas bubble length}

The gas bubble length for a constant superficial gas velocity at varying liquid velocities depends on the ratio of the flow rates as well as on the inlet design. In Fig. 2, the measured gas bubble lengths as well as their distribution are plotted versus the volumetric flow ratio $\left(\epsilon_{\mathrm{L}}^{*}=\right.$ $\dot{V}_{\mathrm{L}} /\left(\dot{V}_{\mathrm{L}}+\dot{V}_{\mathrm{G}}\right)$ for all configurations with an $90^{\circ}$-inlet. In order to compare the values of different reactor widths, we divided the gas bubble length by the corresponding reactor width. For statistical reasons, at least 50 gas bubbles had to be taken into account. Note that gas bubbles with a length of more than $1,300 \mu \mathrm{m}$ exceeded the field of view and could not be detected by our optical system. 


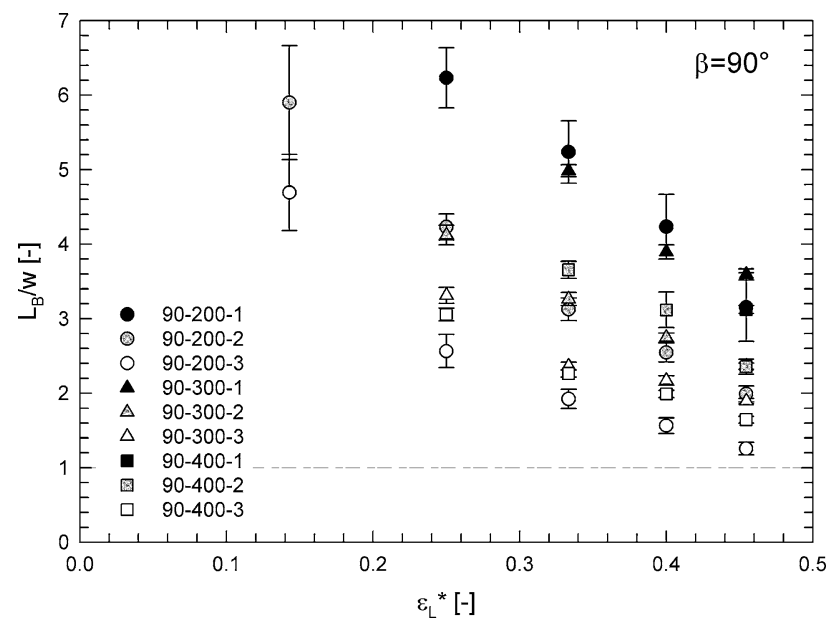

Fig. 2 Measured gas bubble lengths for all $90^{\circ}$-inlet configurations versus the volumetric flow ratio. The line indicates a bubble length equal to the channel width. Same tendencies were observed for $45^{\circ}$ and $135^{\circ}$

In general, increasing the liquid flow rate at constant gas flow rates leads to shorter gas bubbles. The dimensionless gas bubble length, $L_{\mathrm{B}} / w$, is constant within the measurement uncertainties at constant flow rates. If the gas inlet diameter is smaller than the main channel, the gas bubbles are shorter compared with a ratio of $\gamma=1$. Furthermore, the gas bubble length has less variation in length in this case for $\gamma>1$, which is indicated by a significantly smaller standard deviation. The coefficient of variation (=standard deviation/mean value) never exceeds $10 \%$ of the gas bubble length and is in the range of 5\% for $90 \%$ of all measurements performed in these studies. Increasing the inlet angle leads to decreased gas bubble lengths. For constant flow rates, the influence of the inlet angle is less important compared with the influence of the inlet ratio. Furthermore, the stability is affected by the inlet angle: at a constant ratio $\gamma$, inlet angles of $90^{\circ}$ result in a more constant gas bubble length than inlet ratios of $45^{\circ}$ and $135^{\circ}$. This effect diminishes if the width of the gas inlet is significantly smaller compared with the width of the main channel. Figure 3 illustrates the measured gas bubble lengths depending on the volumetric flow ratios $\epsilon_{\mathrm{L}}^{*}$ for different inlet ratios $\gamma$ and inlet angles $\beta$. Values for a reactor width of 300 and $400 \mu \mathrm{m}$ give the same results. For a better visualization of the results the standard deviation, which has the same values as described earlier, is not plotted in Fig. 3.

The reason for the decreasing gas bubble lengths at increasing inlet angles $\beta$ might be caused by the interfacial pressure: as pointed out by Garstecki et al. (2006), the neck of the dispersed phase diminishes to a minimal value where the breakup occurs. Since the interfacial pressure increases with decreasing radii, a faster thinning of the neck results

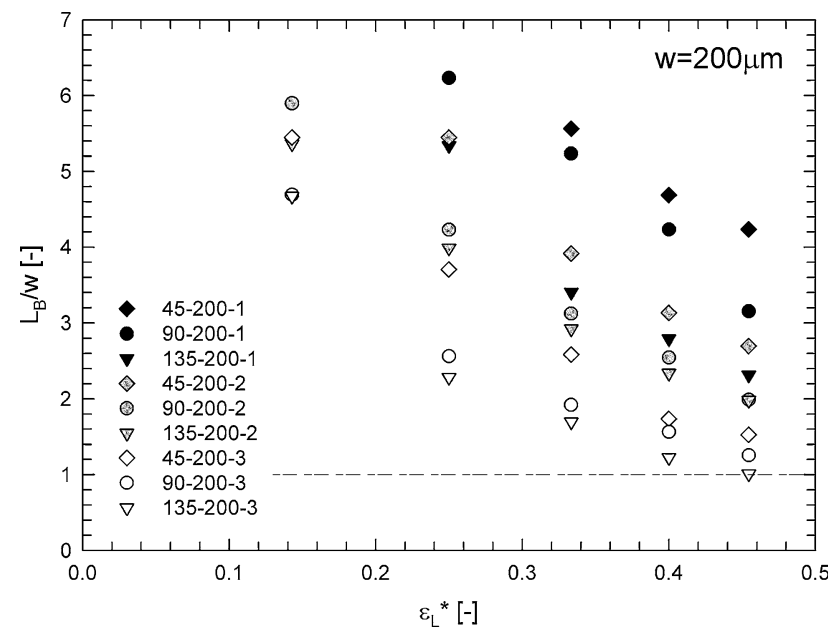

Fig. 3 Measured gas bubble lengths for a width of $200 \mu \mathrm{m}$. The line indicates a bubble length equal to the channel width

in a faster breakup. This thinning was documented in Garstecki et al. (2006) for $\beta=90^{\circ}$ : at the beginning, the width is equal to the width of the gas inlet. When the incoming gas covers the complete channel width, the gas bubble is deflected in the main channel in flow direction due to the incoming liquid. The width of the neck decreases approximately to the half of the inlet width and remains at this value for a certain time. As the pressure due to the incoming liquid increases, the width finally decreases sharply until breakup and the gas flow is interrupted.

In Fig. 4 instantaneous images of the gas bubble formation are given for $\beta=135^{\circ}$ and $\beta=45^{\circ}$. For $\beta<90^{\circ}$ the gas flows towards the liquid inlet. Due to the initial flow direction of the gas phase, the initial width at the neck will be larger than that for a $\beta=90^{\circ}$ inlet; therefore, the interfacial pressure is smaller. Since the neck width reaches its critical value for the breaking off later, the gas bubbles are longer. The same is valid for $\beta>90^{\circ}$ : since the gas flow has the same direction as the incoming liquid, the gas bubble moves toward the flow direction. The thinnest position of the gas bubble moves into the main channel, resulting in a faster breakup ("shearing") and therefore in shorter gas bubbles. This breakup mechanism also explains the decreased gas bubble length for increased $\gamma$. Since the initial total width at the neck is smaller, the critical width causes a faster breakup.

In order to predict the gas bubble length, we have modified Eq. 3. Based on literature, $\alpha$ is assumed to be close to one. Since $\alpha$ strongly depends on the inlet geometry, we need to find a correlation to fit this geometrical value. Starting with

$\alpha=f(\gamma, \beta)=a \gamma^{b} \beta^{c}$

and using the least square fit method, we obtained the following values with a correlation coefficient of 0.96 : 
Fig. 4 Gas bubble breakup for different inlet angles. Volumetric gas flow rate $=0.3 \mathrm{ml} / \mathrm{min}$, volumetric liquid flow rate $=0.1 \mathrm{ml} / \mathrm{min}$
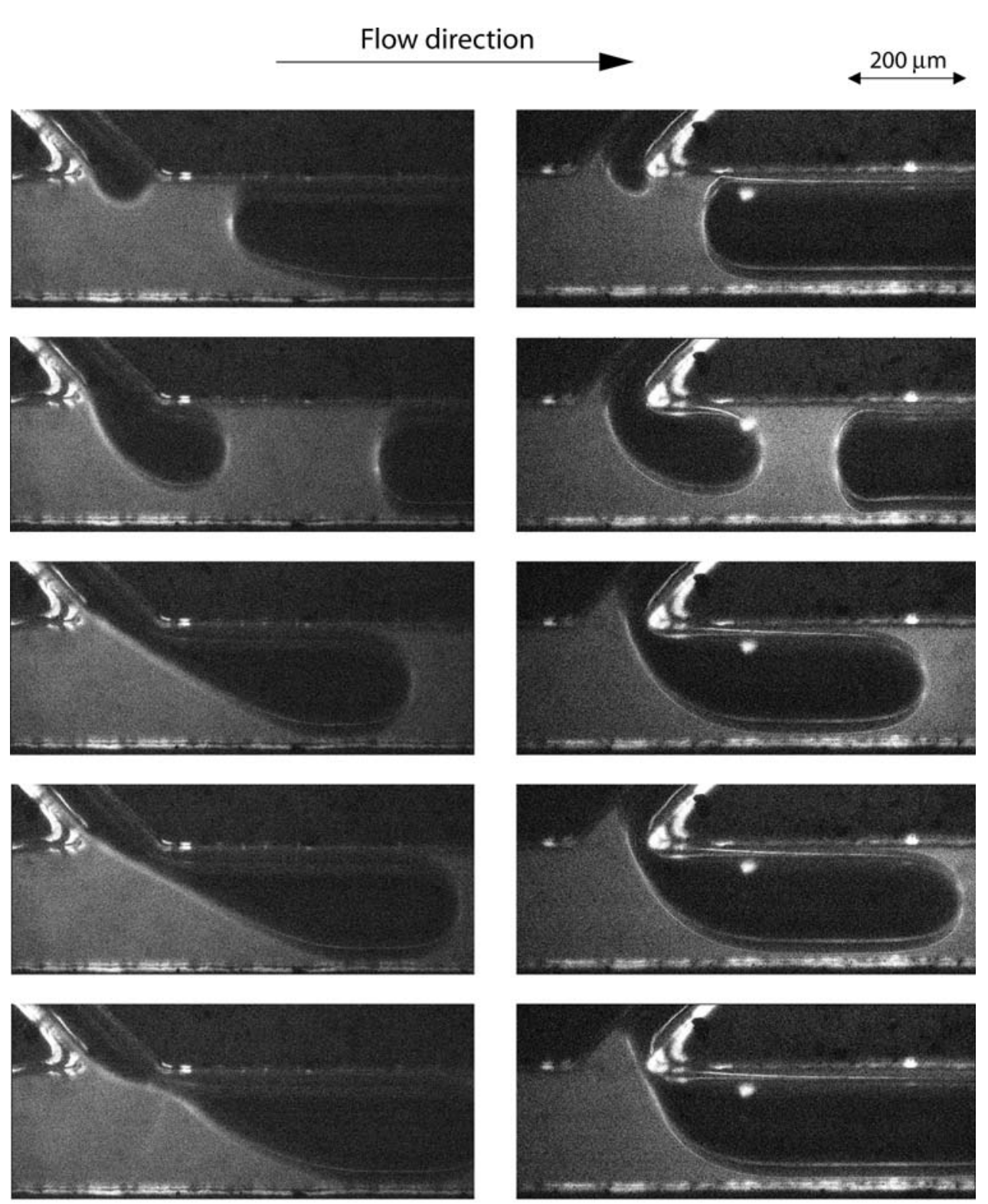

(a) Reactor 135-200-3

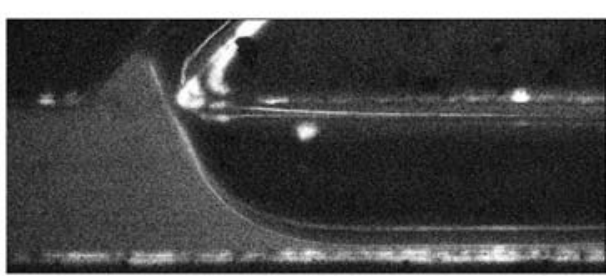

(b) Reactor 45-200-3

$$
\begin{aligned}
& a=17.2131 \\
& b=-1 \\
& c=-0.5
\end{aligned}
$$

Using the geometrical design parameters from Garstecki et al. (2006), $\alpha$ can be calculated to $\alpha=0.91$, which is close to the assumed value of $\alpha=1$. The discrepancy might be caused by different aspect ratios and the measurement uncertainties.

In Fig. 5 the measured values for the dimensionless gas bubble length are plotted versus the values predicted by Eq. 9. Taking the measurement uncertainties into account, we state that the model matches experiment satisfactorily. Therefore, at a constant geometry, the gas bubble length increases with an increasing ratio of gas to liquid flow rate.

\subsection{Liquid slug length}

Since pressure drop and mass transfer strongly depend on the liquid slug length, detailed knowledge on the resulting slug length is mandatory. In Fig. 6, the measured dimensionless slug lengths are plotted versus the volumetric flow ratio $\epsilon_{\mathrm{L}}^{*}$ for all inlets with $\beta=90^{\circ}$. The volumetric flow rates were kept constant so that the corresponding velocities of the flow are higher for small channels.

As for the gas bubble length divided by the channel width, the dimensionless liquid slug length depends for a 


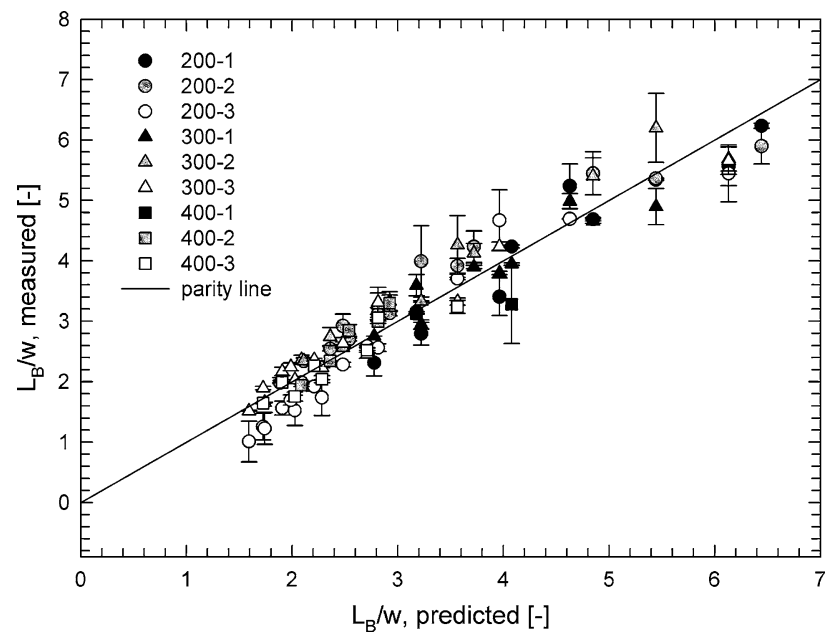

Fig. 5 Dimensionless gas bubble length: model of Eq. 9 versus experiment

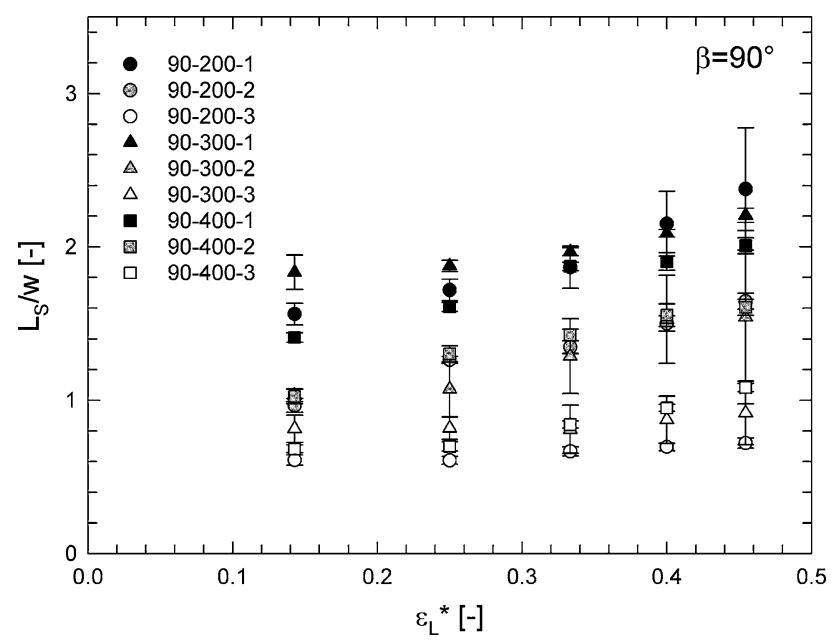

Fig. 6 Dimensionless slug length for all reactors with a $90^{\circ}$ inlet

constant inlet angle of $\beta=90^{\circ}$ on $\epsilon_{\mathrm{L}}^{*}$ as well as on the inlet ratio $\gamma$. Increasing the liquid flow rate compared to the total flow rate results in an increase of the liquid slug length, which corresponds to literature results. If the width of the gas inlet is smaller than the width of the main channel, the liquid slug length decreases. This is the same trend as it was observed for the gas bubble length: for a given inlet angle and flow rate, an inlet design with increasing $\gamma$ leads to decreasing unit cell lengths.

In Fig. 7 the influence of the inlet angle for a given channel width and different inlet ratios is plotted versus the volumetric flow ratio. For illustration reasons the standard deviation was left out. Note that in most experiments the measured standard deviation is below 5\%. For all inlet ratios and $\epsilon_{\mathrm{L}}^{*}$, increasing $\beta$ results in decreasing liquid slug lengths, whereas this effect overlaps with the influence of $\gamma$. The reason for this observed effect can be found in the

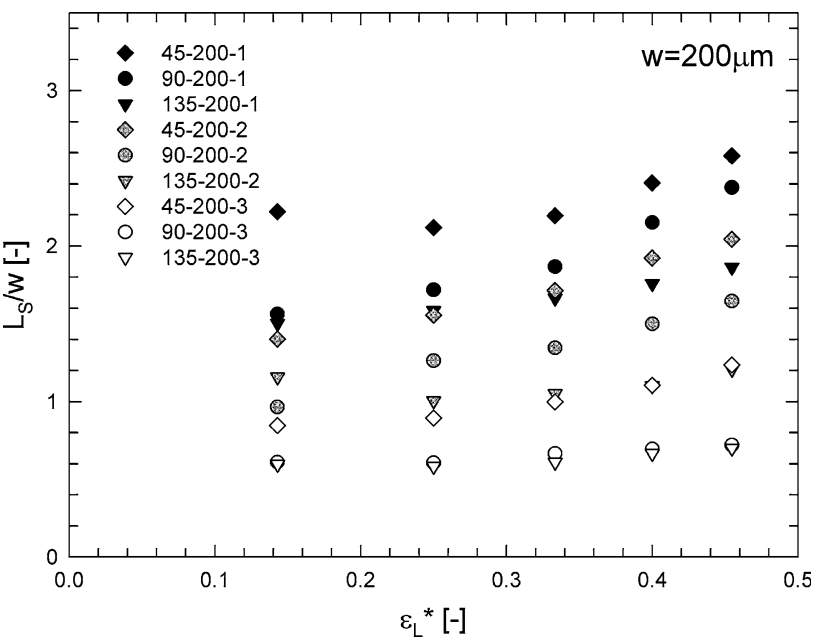

Fig. 7 Dimensionless slug length for all reactors with $w=200 \mu \mathrm{m}$

interfacial pressure. As described earlier, inertia of the inflowing gas affects the width of the gas bubble at the neck and thus the breakup mechanism: if the gas bubbles are short due to the geometry at given flowrates, less liquid is hindered to flow into the channel and thus the liquid pressure is not as high as for long gas bubbles. Therefore, the momentum of the flowing liquid is smaller resulting in a slower initial velocity and thus in shorter liquid slug lengths.

For all geometries, an increase of the liquid flow rate (and therefore of $\epsilon_{\mathrm{L}}^{*}$ ) results in an increase of the liquid slug length. If the ratio of the main channel to the gas inlet channel width increases, the liquid slug length decreases. The standard deviation of the liquid slug length is affected by the inlet ratio $\gamma$; small values of $\gamma$ lead to greater deviations in the liquid slug length. If $\gamma=1$, the dimensionless liquid slug length is in the range of 2-3 for all investigated channel diameters. For $\gamma=2$, the slug length is 1 to 2 times of the hydraulic diameter, whereas for $\gamma=3$ slug lengths in the range of the hydraulic diameter were observed.

Based on Figs. 6 and 7, the dimensionless liquid slug length depends on $\epsilon_{\mathrm{L}}^{*}, \beta$ and $\gamma$. As known from literature, the surface, viscous and inertia forces, expressed by

$\frac{R e}{C a}=\frac{\text { inertia } \quad \text { forces } \times \text { surface } \text { forces }}{{(\text { viscous forces })^{2}}^{2}}$

have a significant influence on the liquid slug length. Correlating

$\frac{L_{\mathrm{S}}}{w}=k \cdot l \cdot n\left[\left(\frac{R e}{C a}\right)^{0.33} \frac{\epsilon_{\mathrm{L}}^{*}}{\gamma^{l} \beta^{m}}\right]+n$

and using the least square method, we obtained 
$k=0.369$

$l=2.5$

$m=1$

$n=3.15$

The measured dimensionless liquid slug lengths as well as the results of Eq. 11 are plotted in Fig. 8. The correlation coefficient between the measured and the predicted data is 0.94. Taking the measurement uncertainties into account, the correlation describes the experimental data for the investigated range of $\epsilon_{\mathrm{L}}^{*}=0.1, \ldots, 0.5$ well.

Obviously, an increase of $\epsilon_{\mathrm{L}}^{*}$ (while keeping all other parameters constant) results in an elevated slug length by factor $\left(\ln \epsilon_{\mathrm{L}}^{*}\right)$. Since $\epsilon_{\mathrm{L}}^{*}$ takes values between 0 and 1 , the increase due to changes in $\epsilon_{\mathrm{L}}^{*}$ can be assumed to be linear. Changes of $\gamma$ are also very significant: increasing $\gamma$ from 1 to 2 leads to a decrease of the dimensionless slug length by $33 \%$. In our experiment the factor $(\mathrm{Re} / \mathrm{Ca})$ was in the order of 1,000 and influences the slug length: increasing inertia forces caused by increased flow rates or the density of the liquid used leads to increased liquid slug lengths. A decrease of the surface tension forces which can be realized using surfactants results in a decreased liquid slug length. Increasing the viscosity while keeping all other parameters constant leads to decreasing liquid slug lengths.

\subsection{Liquid holdup and unit cell length}

An important parameter of Taylor flow is the dimensionless unit cell length $\left(L_{\mathrm{S}}+L_{\mathrm{B}}\right) / w$. In Fig. 9 the dimensionless unit cell length is plotted for all designs with a $90^{\circ}$-inlet versus $\epsilon_{\mathrm{L}}^{*}$. The unit cell length increases with decreasing $\epsilon_{\mathrm{L}}^{*}$ and increases proportionally with the channel

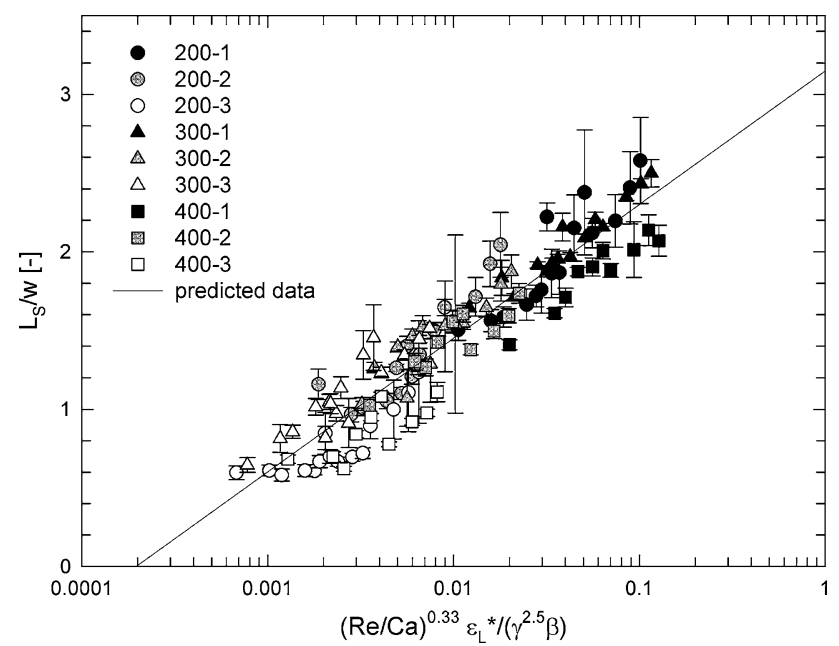

Fig. 8 Dimensionless slug length for all reactors versus (Re/ $C a)^{0.33}(\operatorname{Re} / C a)^{0.33} \epsilon_{\mathrm{L}}^{*} /\left(\gamma^{2.5} \beta\right)$

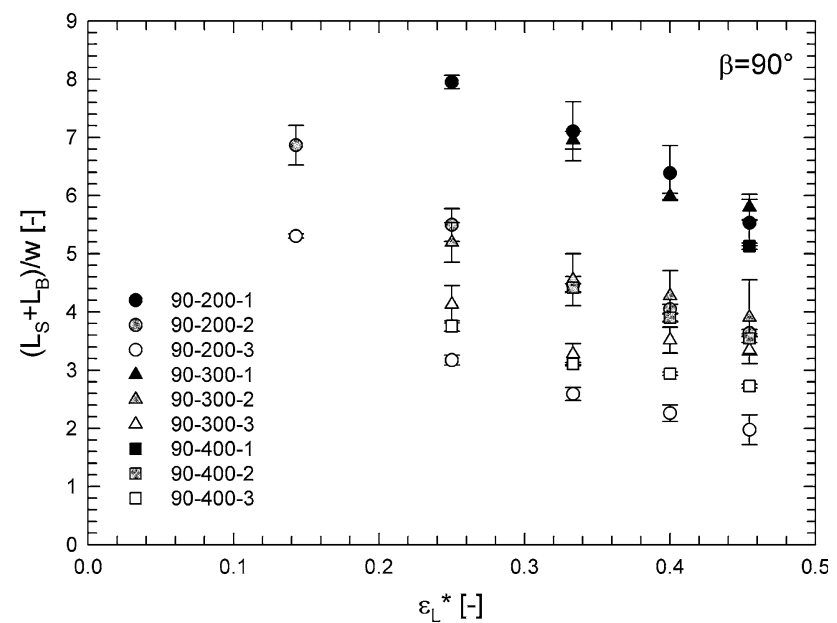

Fig. 9 Dimensionless unit cell length for $\beta=90^{\circ}$

width. A decrease of the unit cell length and thus enhanced mass transfer can be achieved by decreasing the gas inlet width at a constant channel width.

The influence of the inlet angle is not plotted, but according to the results earlier we state as follows: if the gas flow is injected into the main channel in the same direction as the two-phase flow occurs, the unit cell length decreases.

The ratio of gas bubble to liquid slug length versus $\epsilon_{\mathrm{L}}^{*}$ is plotted in Fig. 10 for all inlet configurations with $\beta=90^{\circ}$. The ratio is enhanced compared with the ratio of the corresponding flow rates, which is most pronounced for a channel width of $w=400 \mu \mathrm{m}$ and less important for $w=200 \mu \mathrm{m}$. This is due to the two-dimensional analysis of a three-dimensional shape: since the bubble front as well as the bubble tail consist of a ellipsoid-like shape, whose detailed volume is difficult to determine (starting with

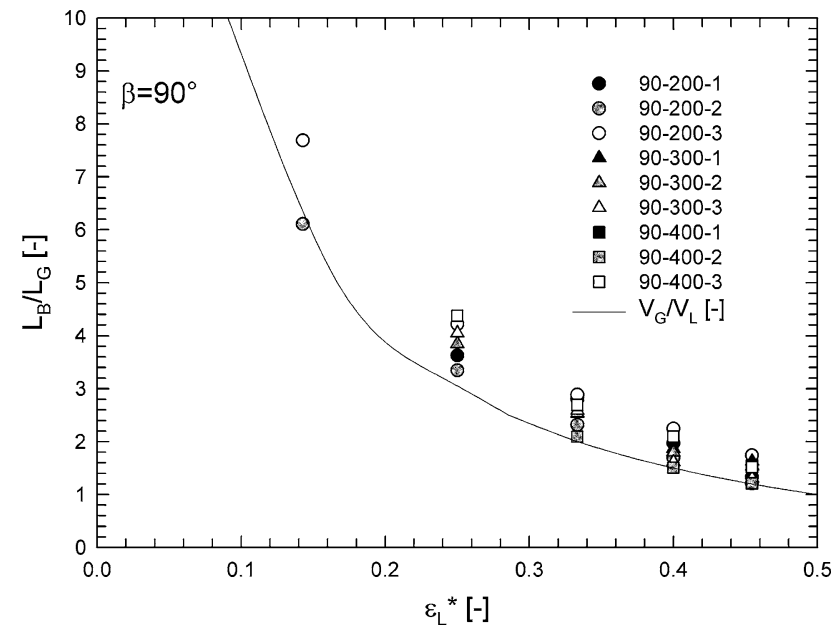

Fig. 10 Dimensionless unit cell length for $\beta=90^{\circ}$ 


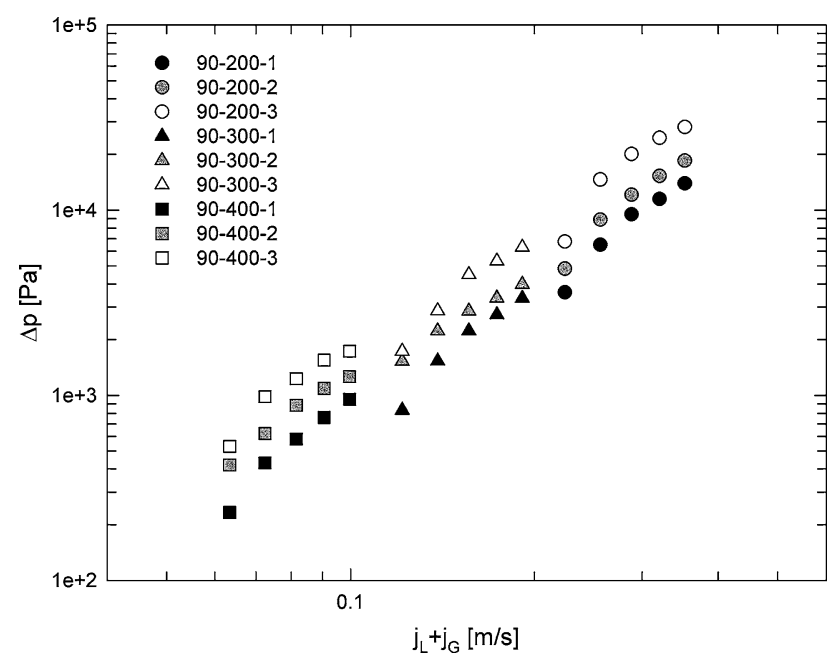

Fig. 11 Measured pressure drop for all $90^{\circ}$-designs versus the total superficial velocity

rotational cross section at the front to a rectangular cross section), we plotted only the maximum bubble length and minimum slug length.

\subsection{Pressure drop}

In Fig. 11 the measured pressure drop for the $90^{\circ}$ designs is plotted versus the sum of the liquid and gas superficial velocity. The pressure drop is influenced by the superficial velocity and the ratio of the gas inlet $\beta$. The influence of the channel width can be neglected, because all measured data points show a straight line for all investigated channel widths at the corresponding gas inlet. Due to an increase in $R e$, the pressure drop increases with increasing total superficial velocity. For the same volumetric flow rates in the gas and in the liquid phase, the pressure drop increases with decreasing channel width and thus an increasing $R e$. Note that the design of the gas inlet influences the pressure drop: $\Delta p_{\gamma=1}<\Delta p_{\gamma=2}<\Delta p_{\gamma=3}$. This is mainly due to the resulting liquid slug length.

The gas-liquid pressure drop in microchannels consists mainly of a frictional pressure drop and can be calculated by

$\Delta p_{\text {fric }}=f \rho_{\mathrm{L}}\left(j_{\mathrm{L}}+j_{\mathrm{G}}\right)^{2} \frac{2}{d_{\mathrm{h}}} \epsilon_{\mathrm{L}} L_{\mathrm{R}}$,

whereas the friction factor $f$ is determined by Kreutzer et al. (2005b) as

$f=\frac{16}{R e}\left[1+a \frac{d_{\mathrm{h}}}{L_{\mathrm{S}}}\left(\frac{R e}{C a}\right)^{0.33}\right]$

The parameter $a$ was found to be dependent on the flow conditions and was found to be $a=0.17$ for a circular capillary with $d_{\mathrm{h}}=2.3 \mathrm{~mm}$ and $R e<50$. In our case

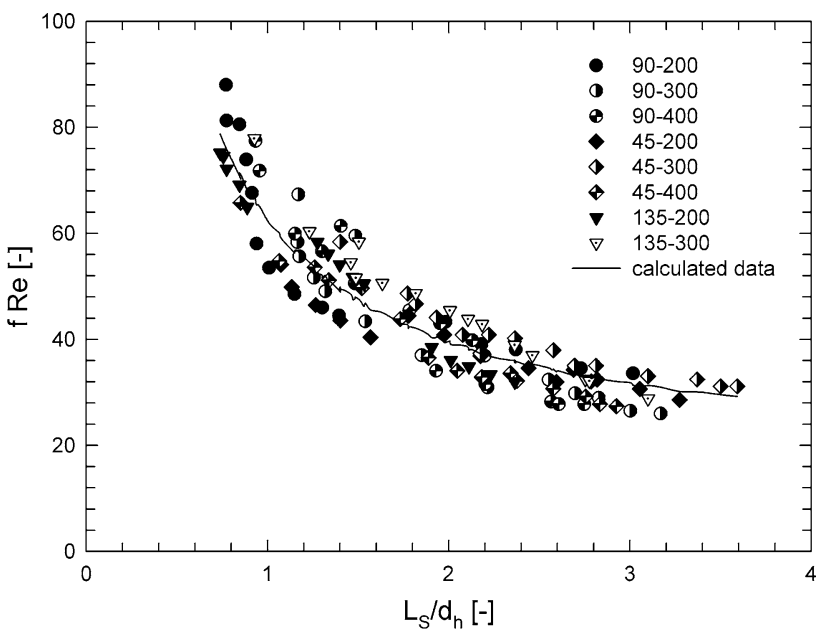

Fig. $12(f R e)$ as a function of the dimensionless slug length for all designs. Points are the measured values, the dotted line represents the calculated friction factor according to Eq. 16

(rectangular PDMS-glass microchannels), the least square method gave $a=0.225$ for $w=200 \mu \mathrm{m}, a=0.21$ for $w=300 \mu \mathrm{m}$ and $a=0.185$ for $w=400 \mu \mathrm{m}$. The decrease of $a$ for an increasing channel width corresponds to the influence of the wall effects. The correlation coefficient amounts 0.98. In Fig. 12 the pressure drop is plotted versus the dimensionless slug length for all reactors designs. For the experimental data, $f$ was calculated from the measured pressured drop using Eq. 16. With the adapted $a$ for different channel widths a dependence of the friction factor of the liquid slug length is visible. Liquid slug lengths in the range of $2-3 d_{\mathrm{h}}$, obtained by $\gamma=1$, result in a small frictional pressure drop compared with short liquid slug lengths in the range of $d_{\mathrm{h}}$ and less. This is valid for all channel widths. Note that $f \cdot R e$ depends only on $L_{\mathrm{S}} / d_{\mathrm{h}}$, independent of the geometry. Since all experiments were performed at the same flow rates, $L_{S} / d_{\mathrm{h}}$ obviously depends only on the flow rate and on $\beta$. According to Kreutzer et al. 2005b) the single phase limit of $f R e=16$ is obtained asymptotically for $L_{\mathrm{S}} / d_{\mathrm{h}}>20$. Since we never obtained such large liquid slug length in our experiments, we cannot validate this value experimentally; nevertheless, extrapolating our measurements results supports this information.

\section{Conclusion}

In this article we discussed the influence of the gas inlet on gas-liquid flow characteristics in rectangular microchannels. Varying the ratio of the gas inlet width compared to the main channel width as well as changing the angle of the gas inlet showed different characteristics of the gas-liquid flow. While the gas-slug ratio is not affected by the 
geometry at a given volumetric flow ratio, the lengths of the gas bubble as well as of the liquid slug and thus the pressure drop change with the geometry. We observed variations of the unit cell length for a given flow ratio of factor 4 .

If the gas phase is injected in the flow direction of the liquid phase $\left(\beta<90^{\circ}\right)$, gas bubbles are shorter than that in the contradictious case. This observation can be explained using the interfacial pressure at the breakup point. Decreasing the gas inlet width at a constant main channel width also results in decreased gas bubble lengths.

The liquid slug length was found to increase with increasing liquid flow rates at constant gas flow rates. If the gas inlet has a smaller width than the main channel, the liquid slug length is shorter compared to a gas inlet with the same width as the main channel. At a constant flow rate and constant $\gamma$, the liquid slug length divided by the channel diameter is constant.

Increasing $R e$ either by increasing the flowrate and/or by decreasing the channel diameter results in an increasing pressure drop. Due to the recirculation in the slug, the pressure drop is also correlated to the slug length. Since the liquid slug length varies with the inlet geometry, the pressure drop increases with smaller gas inlets. With a small adjustment of one parameter, necessary due to the enhanced influence of wall roughness in microchannels, pressure drop correlation obtained for circular minichannels describe the experimental results of this study sufficiently.

This study demonstrates, that enhanced mass transfer within the liquid slugs by a short slug length $\left(S h=f\left(L_{S}\right)\right.$ $w)$ ) can be obtained by injecting the gas phase in the flow direction of the main channel and to build a gas inlet with a smaller channel width than the one of the main channel.

Acknowledgments This work was supported by the Emil-Barell foundation.

\section{References}

Akbar MK, Ghiaasiaan SM (2006) Simulation of taylor flow in capillaries based on the volume-of-fluid technique. Ind Eng Chem Res 45(15):5396-5403
Bercic G, Pintar A (1997) The role of gas bubbles and liquid slug lengths on mass transport in the taylor flow through capillaries. Chem Eng Sci Gas Liquid Solid React Eng 52(21-22):3709_ 3719

Garstecki P, Fuerstman M, Stone H, Whitesides G (2006) Formation of droplets and bubbles in a microfluidic t-junctionscaling and mechanism of break-up. Lab Chip 6(3):437-446

Heiszwolf JJ, Kreutzer MT, van den Eijnden MG, Kapteijn F, Moulijn JA (2001) Gas-liquid mass transfer of aqueous taylor flow in monoliths. Catal Today 69(1-4):51-55

King C, Walsh E, Grimes R (2007) Piv measurements of flow within plugs in a microchannel. Microfluid Nanofluid 3(4):463-472

Kreutzer MT, Kapteijn F, Moulijn J, Heiszwolf J (2005a) Multiphase monolith reactors: chemical reaction engineering of segmented flow in microchannels. Chem Eng Sci 60(22):5895-5916

Kreutzer MT, Kapteijn F, Moulijn JA, Kleijn CR, Heiszwolf JJ (2005b) Inertial and interfacial effects on pressure drop of taylor flow in capillaries. AIChE J 51(9):2428-2440

Laborie S, Cabassud C, Durand-Bourlier L, Laine JM (1999) Characterisation of gas-liquid two-phase flow inside capillaries. Chem Eng Sci 54(23):5723-5735

Liu H, Vandu CO, Krishna R (2005) Hydrodynamics of taylor flow in vertical capillaries: flow regimes, bubble rise velocity, liquid slug length, and pressure drop. Ind Eng Chem Res 44(14):48844897

McDonald JC, Whitesides G (2002) Poly(dimethylsiloxane) as a material for fabricating microfluidic devices. Accounts Chem Res 35(7):491-499

van Steijn V, Kreutzer MT, Kleijn CR (2007) [mu]-piv study of the formation of segmented flow in microfluidic t-junctions. Chem Eng Sci 62(24):7505-7514

Tice JD, Song H, Lyon A, Ismagilov R (2003) Formation of droplets and mixing in multiphase microfluidics at low values of the reynolds and the capillary numbers. Langmuir 19(22):91279133

Waelchli S, Rudolf von Rohr P (2006) Two-phase flow characteristics in gas-liquid microreactors. Int J Multiph Flow 32(7):791-806

Yawalkar AA, Sood R, Kreutzer MT, Kapteijn F, Moulijn JA (2005) Axial mixing in monolith reactors: effect of channel size. Ind Eng Chem Res 44(7):2046-2057

Zheng D, Che D (2006) Experimental study on hydrodynamic characteristics of upward gas-liquid slug flow. Int J Multiph Flow 32(10-11):1191-1218 